\title{
DG-FEM solution for nonlinear wave-structure interaction using Boussinesq-type equations
}

\author{
Allan P. Engsig-Karup ${ }^{1}$, Jan S. Hesthaven ${ }^{2}$, Harry B. Bingham ${ }^{1}$, and \\ Warburton, T. ${ }^{3}$ \\ 1 Technical University of Denmark, Lyngby, Denmark. \\ 2 Brown University, Providence, USA. \\ ${ }^{3}$ Rice University, Houston, USA.
}

December 15, 2006.

\begin{abstract}
We present a high-order nodal Discontinuous Galerkin Finite Element Method (DG-FEM) solution based on a set of highly accurate Boussinesq-type equations for solving general water-wave problems in complex geometries. A nodal DG-FEM is used for the spatial discretization to solve the Boussinesq equations in complex and curvilinear geometries which amends the application range of previous numerical models that have been based on structured Cartesian grids. The Boussinesq method provides the basis for the accurate description of fully nonlinear and dispersive water waves in both shallow and deep waters within the breaking limit. To demonstrate the current applicability of the model both linear and nonlinear test cases are considered where the water waves interact with bottom-mounted fully reflecting structures. It is established that by simple symmetry considerations combined with a mirror principle it is possible to impose weak slip boundary conditions for both structured and general curvilinear wall boundaries while maintaining the accuracy of the scheme. As is standard for current high-order Boussinesq-type models, arbitrary waves can be generated and absorbed in the interior of the computational domain using a flexible relaxation technique applied on the free surface variables.
\end{abstract}

Keywords: Discontinuous Galerkin finite element method, wave-structure interaction, high-order Boussinesq-type equations, nonlinear and dispersive water waves, unstructured and curvilinear grids, grid adaption.

\section{Introduction}

Boussinesq-type equations are in widespread use by coastal engineers for the numerical simulation of nonlinear wave motion in near-shore regions. Up to certain limits in dispersion these equations capture all the important wave phenomena including diffraction, refraction, nonlinear wave-wave interactions and interaction with bottom-mounted structures in regions where viscous and turbulence effects can be neglected. The main idea behind these equations is to reduce the three dimensional potential flow problem to a two dimensional problem by assuming a polynomial variation in the vertical direction. Thus, Boussinesq-type models have become an important engineering tool for the prediction of a range of wave phenomena critical to the design of, e.g., offshore 
windmill foundations, harbours, and exposed loading facilities. A large number of Boussinesq-type models are in use today, and a comprehensive review of the history of Boussinesq theory can be found in Madsen and Schäffer (1999).

Building upon the previous work of Fuhrman et al. (2005) and Engsig-Karup et al. (2006), we focus here on the three-variable formulation due to Madsen et al. (2002), which is one of the most accurate formulations yet developed. In particular, we focus on the Padé $(2,2)$ enhanced version obtained by using the optimized coefficients determined by Jamois et al. (2006), which is accurate in dispersion out to approximately $k d=10$ ( $k$ the wave number and $d$ the water depth) and with an accurate description of the internal flow kinematics to approximately $k d=4$. These equations are thus appropriate for modelling a broad range of water wave phenomena in near-coastal environments. The accuracy and application range of this method exceeds most other Boussinesq-type methods, however it comes at the price of additional variables (degrees of freedom) and more complicated systems of linear equations to solve; and this provides challenges for obtaining efficient numerical solutions.

Many of these challenges have been successfully overcome using a structured (uniformly-spaced, rectangular) grid finite difference solution as reported by Fuhrman et al. (2005) and previous publications cited therein. This finite difference scheme is however at a fixed order of accuracy; and it lacks geometric flexibility both in terms of local grid refinement and the inclusion of other than piecewise rectangular structures. The goal of the present work is to provide a solution with both geometric and order flexibility by means of an unstructured Dicontinuous Galerkin Finite Element Method. We note that the unstructured grid is particularly attractive in terms of spatially resolving realistic geometries and it allows the grid to be adapted to the physical scales of the problem. The latter property can potentially lead to a significant reduction in the computational effort.

Previously, some work has been done in terms of solving Boussinesqtype equations in the framework of finite element methods. The unstructured finite element method (FEM) has been applied to solve various two-variable Boussinesq-type formulations. The classical Boussinesq equations of Peregrine (1967) were solved by Antunes Do Carmo and Seabra Santos (1993), Ambrosi and Quartapelle (1998) and again by Eskilsson and Sherwin (2004). A set of weakly dispersive Boussinesqtype equations was solved and studied by Langtangen and Pedersen (1998). Li et al. (1999) solved the improved Boussinesq equations by Beji and Nadaoka (1996) . The extended Boussinesq equations by Madsen and Sørensen (1992) were solved using a FEM model by Sørensen et 
al. (2004), while the equations of Nwogu (1993) were solved by Walkley and Berzins (1999) and Walkley and Berzins (2002), and Eskilsson and Sherwin, Eskilsson and Sherwin (2003). Recently, Eskilsson and Sherwin $(2002,2005)$ solved the same set of extended equations using a DG-FEM method.

In Engsig-Karup et al. (2006) it was shown that the high-order Boussinesq equations can be solved to high accuracy in one horizontal dimension and with support for unstructured grids using the DGFEM. As a continuation of this previous work, we concentrate here on solutions in two horizontal dimensions. The DG-FEM can handle higher derivatives in the equations and it is relatively easily implemented despite the complexity of the equation system. By this choice of numerical method, we alleviate the problems that are connected with finding a continuous basis with support for higher derivatives and the implications that might result.

The remainder of this paper is organized as follows. Section 2 presents the governing equations of the Boussinesq-type formulation. In Section 3 we consider how to handle wall boundary conditions in a finite computational domain. Section 4 describes the numerical methods chosen for discretizing the governing equations in two horizontal dimensions. In Section 5 we carry out some numerical convergence tests, and finally Section 6 provides some numerical results that have been obtained using the proposed methodology that demonstrate the current applicability of the model.

\section{Governing equations}

A Cartesian coordinate system is adopted with the horizontal $x y$-plane located at the still water level (SWL) and the $z$-axis pointing upwards. The still water depth $d(\boldsymbol{x})$, where $\boldsymbol{x}=(x, y)^{T}$, is measured from the still water level. The fluid domain is everywhere bounded by the sea bed at $z=-d(\boldsymbol{x})$ and the free surface at $z=\eta(\boldsymbol{x}, t)$. The gravitational acceleration $g$ is assumed constant throughout the work and is set equal to $9.81 \mathrm{~m}^{2} / \mathrm{s}$.

The set of Boussinesq equations employed here are derived in the original work of Madsen et al. $(2002,2003)$ and the resulting equations are given here without derivation details.

The evolution of the flow is governed by the exact nonlinear kinematic and dynamic free surface conditions

$$
\partial_{t} \eta=\tilde{w}-\nabla \eta \cdot(\tilde{\boldsymbol{U}}-\tilde{w} \nabla \eta),
$$




$$
\partial_{t} \tilde{\boldsymbol{U}}=-\nabla\left(g \eta+\frac{1}{2}\left(\tilde{\boldsymbol{U}} \cdot \tilde{\boldsymbol{U}}-\tilde{w}^{2}(1+\nabla \eta \cdot \nabla \eta)\right)\right),
$$

with $\tilde{\boldsymbol{U}} \equiv \tilde{\boldsymbol{u}}+\tilde{w} \boldsymbol{\nabla} \eta$ and having introduced the two-dimensional Cartesian gradient operator $\boldsymbol{\nabla}=\left(\partial_{x}, \partial_{y}\right)^{T}$. The solution of the (truncated) Laplace problem for the fluid in the interior along with a kinematic condition at the sea bed $w_{b}=-\boldsymbol{\nabla} d \cdot \boldsymbol{u}_{b}$ is solved in two steps for obtaining the vertical free surface velocity $\tilde{w}$ from

$$
\left[\begin{array}{c}
\tilde{U} \\
\tilde{V} \\
0
\end{array}\right]=\left[\begin{array}{ccc}
\mathcal{A}_{11}-\partial_{x} \eta \cdot \mathcal{B}_{11} & \mathcal{A}_{2}-\partial_{x} \eta \cdot \mathcal{B}_{12} & \mathcal{B}_{11}+\partial_{x} \eta \cdot \mathcal{A}_{1} \\
\mathcal{A}_{2}-\partial_{y} \eta \cdot \mathcal{B}_{11} & \mathcal{A}_{22}-\partial_{y} \eta \cdot \mathcal{B}_{12} & \mathcal{B}_{12}+\partial_{y} \eta \cdot \mathcal{A}_{1} \\
\mathcal{A}_{01}+\mathcal{S}_{01} & \mathcal{A}_{02}+\mathcal{S}_{02} & \mathcal{B}_{0}+\mathcal{S}_{03}
\end{array}\right]\left[\begin{array}{c}
\hat{u}^{*} \\
\hat{v}^{*} \\
\hat{w}^{*}
\end{array}\right],
$$

and then determine $\tilde{w}$ from the solution of one of above using

$$
\tilde{w}=-\mathcal{B}_{11} \hat{u}^{*}-\mathcal{B}_{12} \hat{v}^{*}+\mathcal{A}_{1} \hat{w}^{*} .
$$

It is noted that the set of auxiliary variables $\left(\hat{u}^{*}, \hat{v}^{*}, \hat{w}^{*}\right)$ has been introduced in connection with the employment of Padé approximants to enhance the accuracy of the method. The continuous differential operators characterized by the subscripted letters $\mathcal{A}, \mathcal{B}$, and $\mathcal{S}$ are for brevity all given in Appendix A for the Padé $(2,2)$ formulation, which retains up to third order differential operators.

\section{Boundary conditions}

To close the system of equations given in the former section, we need to impose appropriate boundary conditions at the outer boundaries $\partial \Omega$ of a finite computational domain. At all outer lateral boundaries it is convenient to assume that the computational domain is surrounded by bottom-mounted vertical surface-piercing and fully reflecting walls. By this choice, the wall normal vectors will everywhere be confined to the horizontal plane at the free surface level.

The high-order Boussinesq formulations have been derived from an exact potential flow solution that satisfies the Laplace equation in the interior domain. Characteristic for potential flows are that the streamlines have to be everywhere parallel to the flow and tangential to any solid wall surface. This can be stated by the local slip boundary condition for any fluid particle given as

$$
\boldsymbol{n} \cdot \boldsymbol{u}=n_{x} u+n_{y} v=0, \quad \forall \boldsymbol{x} \in \partial \Omega,
$$

where $\boldsymbol{n}=\left(n_{x}, n_{y}\right)^{T}$ is an outward pointing normal vector, and $\boldsymbol{u} \equiv \boldsymbol{\nabla} \phi$ ( $\phi$ the scalar velocity potential) is a velocity vector in the horizontal 
plane for a fluid particle moving along the wall surface. It is noted that the generalization of this condition to higher order is straightforward as it by standard symmetry considerations is seen to correspond to the homogeneous boundary conditions $\boldsymbol{n} \cdot \nabla^{2 m-1} \phi=0, \forall m=1,2, \ldots$. Assuming that the wall boundary is a streamline and that there is an imaginary flow, a so-called "ghost" flow, on the outside of the wall, we can restate the condition Eq. (5) as a no net flux condition

$$
\boldsymbol{n} \cdot\left(\boldsymbol{u}+\boldsymbol{u}_{g}\right)=0
$$

where $\boldsymbol{u}_{g}$ is a ghost-flow velocity vector for the imaginary flow. Further, we enforce the constraint for the flow in the normal direction by the condition in Eq. (6) and invoke a continuity constraint along the tangential direction by requiring continuity in the size of tangential components

$$
\boldsymbol{t} \cdot \boldsymbol{u}=\boldsymbol{t} \cdot \boldsymbol{u}_{g}
$$

where $\boldsymbol{t}=\left(-n_{y}, n_{x}\right)^{T}$ is a local tangent to the wall. From Eq. (6) and Eq. (7) we obtain the general conditions for arbitrary wall normal vectors which then defines the external ghost flow

$$
u_{g}=\frac{-n_{x}^{2} u+n_{y}^{2} u-2 n_{x} n_{y} v}{n_{x}^{2}+n_{y}^{2}}, \quad v_{g}=\frac{-2 n_{x} n_{y} u+n_{x}^{2} v-n_{y}^{2} v}{n_{x}^{2}+n_{y}^{2}} .
$$

Thus, for general physical boundaries the external ghost flow can be expressed by the internal velocity vector and are therefore coupled to the internal flow field. For the free surface we require that the free surface gradient at the walls is everywhere orthogonal to the wall normal vectors, i.e.

$$
\boldsymbol{n} \cdot \nabla \eta=0 .
$$

Due to the high-order differential operators in the Boussinesq formulation, we need to impose sufficient high-order conditions for a formulation where third-order derivatives are retained to obtain a consistent linear system to solve. It is noted that the same wall conditions for the velocity field found above apply to the auxiliary variables of the Padé-enhanced equation system in Eq. (3).

To be able to simulates waves in generic cases, we would be limiting our scope of possible applications if we were not able to impose radiation boundary conditions. Such boundary conditions are conveniently imposed by the flexible relaxation method due to Larsen and Dancy (1983) in user-defined regions in the interior computational domain. It was shown by Engsig-Karup et al. (2006) that it is an applicable and suitable method for use with unstructured grids. 


\section{Numerical methods}

The governing equations are discretized using a method of lines approach applied to the semi-discrete equation system. A nodal discontinuous Galerkin finite element method (DG-FEM) is used for approximating spatial derivatives. An explicit low-storage $2 N$-RK4 method due to Carpenter and Kennedy (1994) has been used for the temporal integration favoring low storage requirements and a large absolute stability region for testing purposes rather than efficiency, e.g., the classical RK4 method is in general a better choice in terms of efficiency.

For the application of the DG-FEM in arbitrary spatial geometries, the computational domain $\Omega$ is subdivided into $K$ non-overlapping (triangular) elements $\Omega^{k}, \forall k=1, \ldots, K$, such that $\Omega=\cup_{k} \Omega^{k}$. Further, on each element the solution is approximated by a polynomial of at most order $P$, which is constructed using the orthogonal polynomial basis functions originally due to Proriol (1957) defined in a collapsed coordinate system on a standard triangle. By uniqueness of the interpolating polynomials these can be represented using an equivalent nodal Lagrangian basis. Further, by a unique map from physical coordinates to the coordinates of a standard element a quadrature-free implementation is used, see Hesthaven and Warburton (2002). Thus, on each element the solution is approximated by a finite series expansion of the form

$$
u^{k}(\boldsymbol{x})=\sum_{n=1}^{N} u_{n}^{k} l_{n}(\boldsymbol{x}), \quad u^{k}(\boldsymbol{x}) \in L^{2}\left(\Omega^{k}\right),
$$

where the nodal Lagrangian basis functions $l_{n}\left(\boldsymbol{x}_{n}\right) \equiv 1$ and $l_{m}\left(\boldsymbol{x}_{n}\right) \equiv 0$, $\forall m \neq n$, are defined on the optimal (electro-static) nodal distribution due to Hesthaven (1998) and $N$ is the (fixed) number of nodes within each element.

The method due to Bassi and Rebay (1997) represents a straightforward central discretization method, and is therefore used as a convenient and practical first choice for representing all spatial differential operators discretely. By this approach high-order spatial operators are decoupled into a set of coupled first order equations and then after employing a DG-FEM discretization procedure, they are subsequently reduced to single operators by local elimination of the introduced auxiliary variables.

Applying the same general discretization procedure used by EngsigKarup et al. (2006), the free surface equations Eq.'s (1)-(2) are restated for each element $\Omega^{k}$ using a strong DG-FEM procedure defined on a 
Cartesian grid as follows

$$
\begin{aligned}
\int_{\Omega_{k}} v(\boldsymbol{x}) \partial_{t} \eta d \boldsymbol{x}^{k} & =\int_{\Omega_{k}} v(\boldsymbol{x}) Q(\boldsymbol{x}) d \boldsymbol{x}^{k} \\
\int_{\Omega_{k}} v(\boldsymbol{x}) \partial_{t} \tilde{\boldsymbol{U}} d \boldsymbol{x}^{k} & =-\int_{\Omega_{k}} v(\boldsymbol{x}) \boldsymbol{\nabla} F(\boldsymbol{x}) d \boldsymbol{x}^{k} \\
& +\oint_{\partial \Omega_{k}} v(\boldsymbol{x}) \boldsymbol{n}\left(F(\boldsymbol{x})-F^{*}(\boldsymbol{x})\right) d \boldsymbol{S}^{k}
\end{aligned}
$$

having introduced the auxiliary functions

$$
\begin{aligned}
& Q(\boldsymbol{x})=\tilde{w}-\boldsymbol{\nabla} \eta \cdot(\tilde{\boldsymbol{U}}-\tilde{w} \boldsymbol{\nabla} \eta), \\
& F(\boldsymbol{x})=g \eta+\frac{\tilde{\boldsymbol{U}} \cdot \tilde{\boldsymbol{U}}}{2}-\frac{\tilde{w}^{2}}{2}(1+\boldsymbol{\nabla} \eta \cdot \nabla \eta) .
\end{aligned}
$$

Here $\boldsymbol{n}=\left(n_{x}, n_{y}\right)^{T}$ is an outward pointing (spatially varying) normal vector along the faces of the $k^{\prime}$ th element, $v(\boldsymbol{x})$ is a test function chosen from the same function space used for the approximation of the solution, and $\boldsymbol{S}^{k} \in \partial \Omega^{k}$ is the boundary of the $k^{\prime}$ th element. The auxiliary functions are evaluated by direct products of the interpolant functions at interpolating nodes in the grid. This can lead to aliasing errors, which makes it necessary to adopt a suitable de-aliasing strategy, see Section 4.1.

For the discretization of Eq. (12) the numerical Lax-Friedrichs fluxes are chosen

$$
\boldsymbol{n} \cdot F^{*}\left(\boldsymbol{x}^{-}, \boldsymbol{x}^{+}\right)=\boldsymbol{n} \cdot \frac{F\left(\boldsymbol{x}^{-}\right)+F\left(\boldsymbol{x}^{+}\right)}{2}-0.5|s|\left(\tilde{\boldsymbol{U}}^{+}-\tilde{\boldsymbol{U}}^{-}\right),
$$

to be able to introduce a small amount of numerical dissipation into the scheme for stabilization purposes. Discarding the last term in Eq. (15), i.e. setting $s=0$, results in simple central fluxes, which are used for the approximation of all spatial operators in the following.

The two-step procedure for the Boussinesq system Eq.'s (3)-(4) can be stated in the general form

$$
\begin{aligned}
\boldsymbol{f} & =\mathcal{A} \hat{\boldsymbol{u}}^{*}, \\
\tilde{w} & =\mathcal{B} \hat{\boldsymbol{u}}^{*},
\end{aligned}
$$

with the left hand-side $\boldsymbol{f}=(\tilde{U}, \tilde{V}, 0)^{T}$ and the right hand-side vector $\hat{\boldsymbol{u}}^{*}=\left(\hat{u}^{*}, \hat{v}^{*}, \hat{w}^{*}\right)^{T}$. The definitions for the global $\mathcal{A}$ and $\mathcal{B}$ operators follow from Eq.'s (3)-(4). These global Boussinesq block system operators can be assembled using the discretization method of Bassi-Rebay. To optimize efficiency and simplify the implementation, we choose to apply a collocation approach where the DG-FEM is only used for determining spatial derivatives, and therefore only a small set of global 
discrete differential operators has to be determined for use with the nodal DG-FEM setup.

For a finite domain with two horizontal dimensions, all high-order differential operators can be constructed from the discrete representation of the first order differential operators $\mathcal{D}_{x}^{D}, \mathcal{D}_{x}^{N}, \mathcal{D}_{y}^{D}$ and $\mathcal{D}_{y}^{N}$. For example, the second order differential operators

$$
\mathcal{D}_{x x}^{D}=\mathcal{D}_{x}^{N} \mathcal{D}_{x}^{D}, \quad \mathcal{D}_{y y}^{N}=\mathcal{D}_{y}^{D} \mathcal{D}_{y}^{N},
$$

where boundary conditions have been imposed and the superscripts indicate that either homogenous Dirichlet (D) or Neumann (N) boundary conditions have been imposed along the indicated Cartesian direction at the outer boundaries. For example, consider the approximation of the spatial operator in the normal direction derived for the $k$ 'th element as

$$
\int_{\Omega^{k}} v(\boldsymbol{x}) f d \boldsymbol{x}=\int_{\Omega^{k}} v(\boldsymbol{x}) \partial_{n} u d \boldsymbol{x}+\oint_{\partial \Omega^{k}} v(\boldsymbol{x}) \boldsymbol{n} \cdot\left(u^{*}-u^{-}\right) d \boldsymbol{S} .
$$

This standard discretization result in a discrete scheme of the form given in compact notation using discrete operators as

$$
\left|\mathcal{J}^{k}\right| \mathcal{M} f^{k}=\left(\mathcal{S}_{n}+\mathcal{B}_{n}\right) u^{k}
$$

or simply

$$
f^{k}=\frac{1}{\left|\mathcal{J}^{k}\right|} \mathcal{M}^{-1}\left(\mathcal{S}_{n}+\mathcal{B}_{n}\right) u^{k}=\mathcal{D}_{n}^{D / N} u^{k}
$$

Note that the superscript letters also indicate the symmetry of the variable they should be applied on. By this approach, the high-order differential block operators can be constructed. For example, the 2D operator $\mathcal{A}_{11}$ (which works on the odd-symmetric variable $\hat{u}^{*}$ ) can be approximated by a discrete operator as

$$
\mathcal{A}_{11}^{D}=\mathcal{I}-\alpha_{2} \cdot \mathcal{D}_{x}^{N} \mathcal{D}_{x}^{D}
$$

where $\mathcal{I}$ is the identity matrix of size $D o F$ x $D o F$, with $D o F$ the total number of grid nodes, and $\alpha_{2}$ is a diagonal matrix holding the spatial coefficients at every grid node. However, although this construction procedure is straightforward, we need to be careful.

Using DG-FEM the discrete differential operators do not commute, i.e. $\mathcal{D}_{x} \mathcal{D}_{y} \neq \mathcal{D}_{y} \mathcal{D}_{x}$. Hence, we have to be careful in the choice of discretization for the cross-differential terms. For example, In Eq. (3) the block operator $\mathcal{A}_{2}$ appears symmetrically about the diagonal of the $\mathcal{A}$ operator. Hence, respectively above (U) and below (L) the diagonal of 
$\mathcal{A}$ we mimic the symmetry by the following orders of differentiation

$$
\begin{aligned}
& \mathcal{A}_{2, U}^{D}=-\alpha_{2} \cdot \mathcal{D}_{x}^{N} \mathcal{D}_{y}^{D}, \\
& \mathcal{A}_{2, L}^{D}=-\alpha_{2} \cdot \mathcal{D}_{y}^{N} \mathcal{D}_{x}^{D} .
\end{aligned}
$$

There might be other suitable choices of discretization, however, the above has been found to work well with the Padé $(2,2)$ version of the rotational velocity formulation.

Since we are solving potential flow problems, boundary conditions can be imposed as described in Section 3 using a mirror principle. This is convenient, since all of these operators can be constructed a priori to the actual simulations, and thereby save computational resources during simulations in time. To implement any discrete version in a finite domain of a Boussinesq formulation involving high-order differential operators, requires a careful implementation which takes into account the symmetry properties of the solution near the outer boundaries. As we only consider wall boundary conditions in the present work, we have to make sure that we impose the correct boundary conditions on each of the differential operators, which are dependent on the solution variable it is applied on. For example, the discrete approximation of the spatial operator $\mathcal{B}_{11}$ should be implemented in two different ways; If it is applied to the auxiliary variable $\hat{u}^{*}$ it should have the same outer boundary conditions imposed as the physical free surface velocity field component $\tilde{u}$, in which case it can be discretized as

$$
\mathcal{B}_{11}^{D, x}=\beta_{1} \cdot\left(\mathcal{D}_{x}^{D}\right)-\beta_{3}\left(\mathcal{D}_{x}^{D}\right)\left(\mathcal{D}_{x}^{N} \mathcal{D}_{x}^{D}+\mathcal{D}_{y}^{D} \mathcal{D}_{y}^{N}\right),
$$

and if it is applied to $\hat{w}^{*}$ then as

$$
\mathcal{B}_{11}^{N, x}=\beta_{1} \cdot\left(\mathcal{D}_{x}^{N}\right)-\beta_{3}\left(\mathcal{D}_{x}^{N}\right)\left(\mathcal{D}_{x}^{D} \mathcal{D}_{x}^{N}+\mathcal{D}_{y}^{D} \mathcal{D}_{y}^{N}\right) .
$$

Nonlinear terms are likewise approximated using direct products at the collocation points. For example, the approximation of a nonlinear term can be done as

$$
\partial_{x} \eta \cdot \mathcal{B}_{11} \approx \eta_{x} \cdot \mathcal{B}_{11}^{D, x},
$$

where $\eta_{x}$ is a diagonal matrix which contains the discrete nodal values of $\partial_{x} \eta$. This approach can potentially suffer from aliasing errors. However, it allows for a fast, efficient and uncomplicated way of reconstructing the operators, which is convenient for the fully nonlinear simulations in terms of efficiency.

The current strategy for solving the linear system in Eq. (3) every stage of the time integration is an unrestarted GMRES method due to Saad and Schultz (1986) suitable for unsymmetric systems. To improve 
efficiency we currently use a full $\mathcal{L U}$ factorization of the linearized system matrix as a preconditioner combined with the Symmetric Reverse Cuthill-McKee reordering for reducing the fill-in. This solution strategy has been found to scale within $\mathcal{O}\left(n^{1.5}\right)$ ( $n$ the number of equations in the linear system) and hence only represents a robust algorithmic choice sufficient for demonstrating the applicability of the chosen method. Optimizing the efficiency for scalability of the model in the DG-FEM framework is the subject of ongoing research.

The numerical model has been implemented using the DG-FEM software package Sledge++, see Wilcox (2006).

\subsection{De-Aliasing}

It is well-known that nonlinear PDEs can result in alias-driven errors and possibly instabilities. Therefore to remedy these potential problems, we have employed a local de-aliasing technique which works on the modal coefficients of the Proriol basis of the expansion given in Eq. (10) such that the filtered local solutions are of the form

$$
u^{k}(\boldsymbol{x})=\sum_{n=1}^{N} \sigma\left(n, N_{c}, \alpha, s\right) \hat{u}_{n}^{k} \phi_{n}(\boldsymbol{x}),
$$

where the exponential low-pass cut-off filter is defined as

$$
\sigma\left(i, N_{c}, \alpha, s\right)=\left\{\begin{array}{ll}
1 & , 0 \leq i<N_{c} \\
\exp \left(-\alpha\left(\frac{i-N_{c}}{P+1-N_{c}}\right)^{s}\right), & N_{c} \leq i \leq P
\end{array},\right.
$$

where $i$ is polynomial order of the expansion mode, $N_{c}$ is the cut-off frequency, and $s$ is the order of the filter. By adjusting the parameters appropriately, it is possible with this filter to obtain similar damping profiles as alternative filters and it can therefore conveniently be used for de-aliasing purposes generically.

The local filtering technique has been found to be sufficient for mildly nonlinear cases. However, for extreme nonlinear cases where the simulations may be more prone to aliasing errors, we need alternative stabilization means, and this is a subject of ongoing research.

\section{Convergence tests}

\subsection{Small-Amplitude Standing WaVes}

We seek to test the numerical scheme proposed in the former section. In particular, we seek to demonstrate that the correct slip boundary 


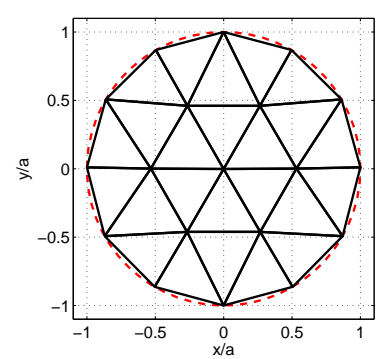

a) Geometric representation of circular domain using $K=24$ triangular elements.

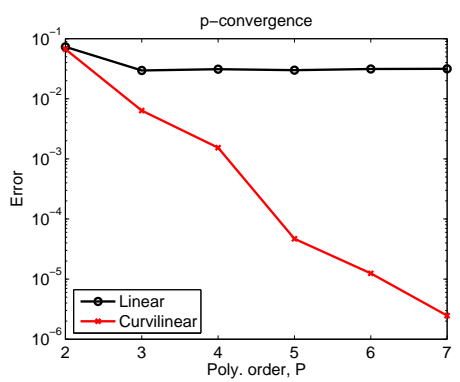

b) p-type convergence test.

Figure 1. Convergence test for a linear standing wave in a circular basin for different boundary type approximations. Boundary approximation using piece-wise linear (full lines) or curvilinear (dotted) element face approximations.

conditions are imposed along the domain wall boundaries. For this purpose we simulate waves in a curvilinear geometry to both test and demonstrate the generality of the chosen discretization.

An analytical solution for linear standing waves in a circular basin of radius $a$ is given at the free surface in polar coordinates as (e.g. see Mei (1989))

$$
\begin{aligned}
\phi(r, \theta, t) & =-\frac{H}{2} c \operatorname{coth}\left(k_{m} d\right) J_{1}(k r) \sin (n \theta) \sin (\omega t), \quad z=0 \\
\eta(r, \theta, t) & =\frac{H}{2} J_{1}\left(k_{m} r\right) \sin (n \theta) \cos (\omega t)
\end{aligned}
$$

where the mode wave number $k_{m}$ is defined from the $m$ 'th zero of $\left.J_{1}^{\prime}(r)\right|_{r=k_{m} a}=0$ with $J_{1}$ the Bessel function, $H$ is the maximum wave height of the oscillation, $c$ is the wave speed given by the linear dispersion relation. The polar coordinates can be transformed to Cartesian coordinates using $x=r \cos (\theta)$ and $y=r \sin (\theta)$.

A linear accuracy test is carried out using the rotational velocity Padé $(2,2)$ model using the curvilinear grid shown in Figure 1 a), and the results are presented in b) for a standing wave defined by $\left(k_{3} a, k_{3} d, n\right)=(5.33,2 \pi, 1)$. The accuracy test demonstrates spectral convergence for simulating the chosen mode after one wave period of time using the curvilinear mesh and having used a sufficiently small time step $\Delta t$ for the spatial errors to dominate. However, if the domain boundary is represented by piece-wise linear boundary approximations then as expected the model fails to converge to the exact solution due to the $\mathcal{O}(h)$ errors made in the geometric representation. 


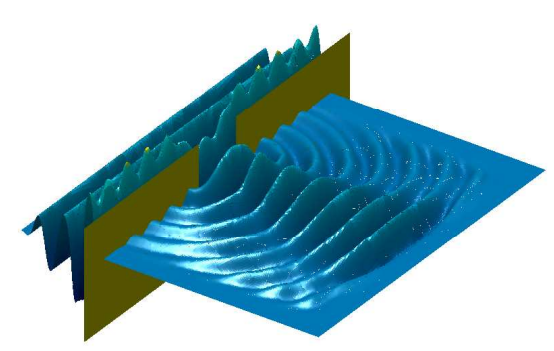

Figure 2. Breakwater gap diffraction of waves. Diffraction function shown for all of the domain including the relaxation regions.

\section{Numerical examples}

We conclude with some numerical examples that demonstrate some of the current capabilities of the model based on the proposed methodology.

\subsection{BREAKWATER GAP DifFraCtion}

The diffraction of water waves near structures is an important phenomenon, and in particular in complex geometries. For example, in connection with the design of harbors or other areas where breakwaters shelter moored ships, serve as coastal protection strategies, etc. In such cases we are interested in predicting calm areas (little energy focusing) or areas which are exposed to significant wave motion due to penetration of waves into such areas of interest. The result of diffraction is that wave fronts "bend" about corners and it is primarily a linear wave phenomenon. Thus, as a step toward modelling water-waves in complex geometries, a classical rigid breakwater gap diffraction test is carried out. Often numerical results are compared to the experimental results from the study by Pos (1984). In this study a generalized breakwater gap configuration was used with splitting plates connected from the wave paddle and extending to the breakwater tips in order to effectively reduce reflections and other secondary effects introduced on the seaward side of the breakwater from the backward side. Experimental and numerical results from a FEM model were later presented by Pos and Kilner (1987).

In numerical studies, linear breakwater gap diffraction problems have been considered using a number of different numerical models based on different model equations in the past. A survey of previous published numerical results, e.g., Panchang and Kopriva (1989), Skot- 


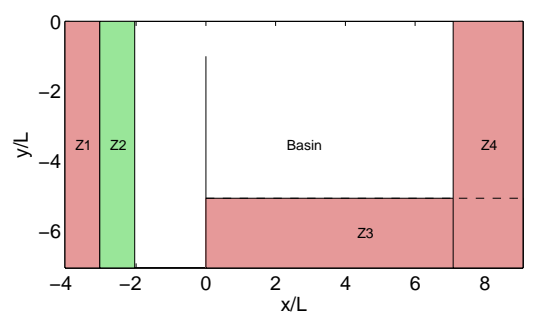

Figure 3. Relaxation zone setup for gap diffraction test.

ner and Apelt (1999), Walkley and Berzins (2002), and Fuhrman et al. (2005) to mention some, observe (often minor) unwanted oscillatory effects in the diffraction solution they obtained. These oscillatory effects are in general attributed to the presence of the (singular) corner point of the breakwater and may also be due to radiation boundary conditions which are not fully absorbing. To remedy numerical instabilities caused by the singular corner, some authors found it necessary to introduce some artificial viscosity in the vicinity of the corner to dampen any unwanted spurious oscillations. The (combined) effects that can be attributed to the breakwater corner point, the reflected waves and the introduction of artificial viscosity seem to be less clear.

In the present study we pursue a slightly different path; we seek to resolve the exact geometry used in the solutions by Penny and Price (1952) as this allows for a direct comparison with the analytical results, and we aim at introducing no artificial damping at all near the breakwater tip.

For the evaluation of the computed results we use the analytical diffraction solutions based on linear theory as described in Penny and Price (1952), which are based on the original work by Sommerfeld (1896). These analytical solutions are based on the assumptions that the monochromatic waves are normally incident on the (infinitely thin) breakwater, where they are completely reflected, the sea bed is uniformly flat and of arbitrary depth, and that the domain is infinitely large to allow the waves to radiate to infinity. Further, it is noted that the break water gap diffraction solution by Penny and Price used for comparison, is a reasonable approximation for relative gap sizes of $b / L>1$, although as noted in Pos and Kilner (1987), it is only strictly accurate for $b / L>2$ due to the approximate boundary conditions for the case of two rigid barriers of the breakwater gap when the gap is small.

As shown in Figure 3, relaxation zones are used to generate and absorb waves in the region Z1 and Z2 at the western boundary each 
zone one wave length in width. Absorbing sponge layers are introduced on the sea side of the breakwater at both the eastern and southern boundaries to fully absorb any outgoing waves over two wave lengths. In the south-east corner the sponge layers overlap, to generate a smooth relaxation down to the corner as well as the domain boundaries. The grid in Figure 4 is generated using DistMesh due to Persson and Strang (2004), and for the infinitely thin wall to be a domain boundary, it was necessary to post-process the generated grid in order to decouple all elements belonging to this internal wall boundary.

Linear sinusoidal incident waves with wave period $T=0.59 \mathrm{~s}$, wavelength $L=0.495 \mathrm{~m}$, wave height $H=0.055 \mathrm{~m}$ are generated. The angular wave frequency is determined using the linear dispersion relations for the Boussinesq equations. The still-water depth throughout the domain is $d=0.125 \mathrm{~m}$ (corresponding to a dimensionless depth $k d=1.3$ ). These waves propagate from the inside of a break water gap of width $b=0.99 \mathrm{~m}$ into the basin. Thus, the breakwater gap to wave length ratio is $b / L=2$ for this case. The waves are generated until $t=25 \mathrm{~s}$, and a time increment of $\Delta t=0.03 \mathrm{~s}$ is used. The computed linear diffraction diagram, determined as the ratio between the computed wave height and the incident wave height $H_{c} / H$, is seen in Figure 5 for a locally adapted grid using local polynomial orders of $P=6$. There is excellent agreement on both sides of the breakwater, however, minor discrepancies are seen in the shadow zone on the seaward side. Local refinement has been used to ensure sufficient spatial resolution to resolve sharp gradients near the breakwater tip, which was found to be a requirement for obtaining an accurate solution. Without local refinement, a number of tests showed a trend where the wave heights were slightly over-estimated near the wall in the shadow zone. As we have introduced no damping such trends can be explained by the numerics of the scheme, possible reflections from relaxation zones and in particular the geometric subdivision of the spatial geometry. In any test carried out, the singular point in the solution at the corner has not been found to be the cause of numerical instability, and this is believed to be a result of the chosen discretization which resembles the analytical properties of the linearized system of equations closely, see Engsig-Karup et al. (2006). However, as seen in Figure 6 the flow vectors at each of the nodes positioned at the connecting corner point have some directional spreading and much larger relative magnitude in comparison with the immediate surrounding flow field. The flow field has been automatically scaled up almost 40 times for the visualization. Fortunately, based on the computed results we conclude that the effect remains local and is caused by the non-unique local normal vectors at the singular nodal point. 


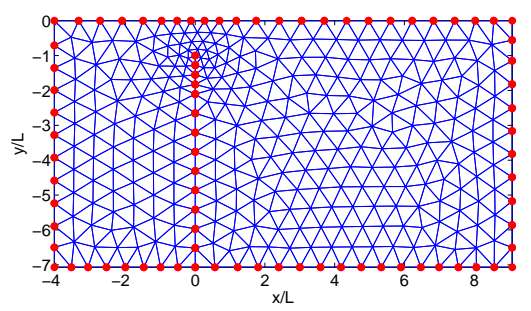

Figure 4. Grid. 599 elements. Vertice nodes belonging to the domain boundary shown with large (red) dots. $b / L=2$.

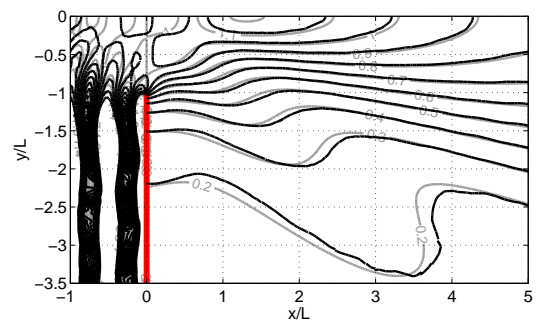

Figure 5. Comparison between exact (gray) and computed (black) linear diffraction solution. $b / L=2$. Waves are generated normal to and at the western boundary.

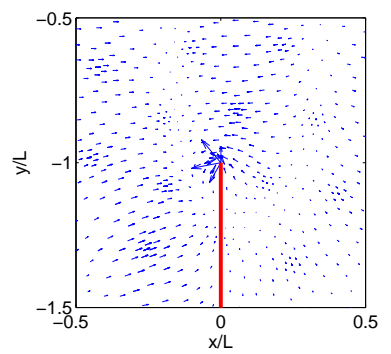

Figure 6. Snapshot of flow field about the singular corner point. $b / L=2$.

\subsection{SCATtering OF LinEAR WAVES ABOUt A VERTICAL CYLINDER IN OPEN WATER}




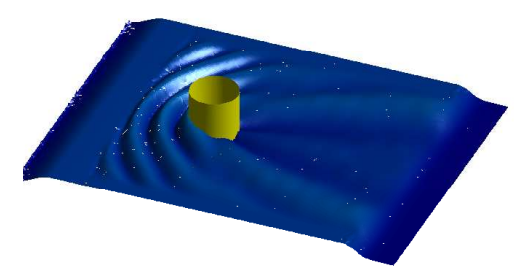

Figure \%. Scattering of waves about a cylinder in open water. Diffraction function shown for all of the domain (relaxation regions also shown).

There are only a few geometries in the theory of water-wave diffraction where exact analytical solutions have been found. For the scattering of a plane incident field of water waves about a vertical cylinder in open water with a flat sea bed, we can use the analytical solution due to McCamy and Fuchs (1954) for finite-depth cases for comparison with our numerical results. Based on the result for the standing wave test in Section 5.1, we choose to approximate the circular cylinder boundary using curvilinear elements since the alternative polygonal representation can result in inaccurate solutions.

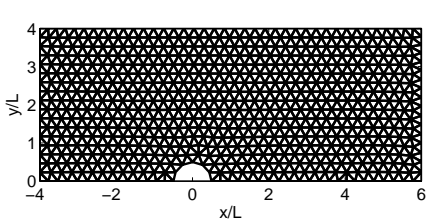

a) Grid. 1261 elements.

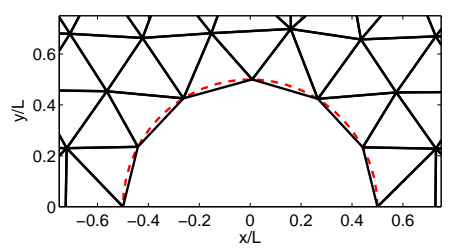

b) Curvilinear approximation (red, dotted).

Figure 8. Grid.

As the problem is symmetric about the horizontal axis, we generate a grid that only represents the upper portion of the plane, to reduce the problem size, see Figure 8. As most of the scattering effects are seen in the near-field of the cylinder, we only seek to resolve the immediate region of the cylinder and allow all scattered waves or the incident waves to be fully absorbed to resemble radiation boundary conditions.

At the Western and Northern boundaries we both generate and absorb waves simultaneously and at the Eastern region we setup a sponge layer for wave absorption. As the total wave field can be considered as a superposition of the incident wave field and a scattered wave field throughout the domain, we generate waves matching the incident wave field at both the Western and Northern boundaries in order to resemble 


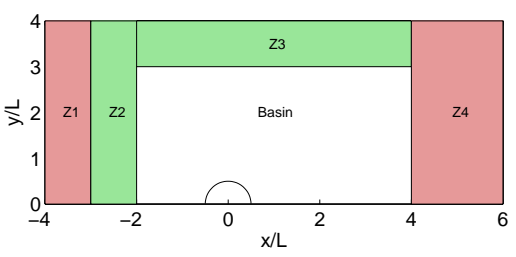

Figure 9. Relaxation zone setup for test of scattering about a cylinder in open water.

the analytical far-field solution as close as possible. See Figure 9. We focus on a single test case to investigate more closely the performance of the model for the case of wave scattering about a cylinder with radius $a=0.5 \mathrm{~m}$. Waves of length $L=1.0 \mathrm{~m}$ are generated over a flat sea bed of still water depth $d=1 /(2 \pi) \mathrm{m}$ such that the dimensionless depth is $k d=1.0$ everywhere in the domain.

In Figure 11 we have shown the computed diffraction diagrams for both the exact linear solution and the computed solution for the case of a curvilinear boundary representation of the solid cylinder surface. The computed results are determined from data obtained at the end of the simulation over (the last) one wave period of time. Final time was $t=25$ $\mathrm{s}$, and the time increment was $\Delta t=0.03 \mathrm{~s}$. A local approximation order of $P=4$ is used.

A qualitative comparison of the results can be made by inspecting Figure 11. The computed results obtained using curvilinear elements locally results in a good agreement with the analytical results. However, some discrepancies caused primarily by reflections from the relaxation zones exists. These discrepancies at the cylinder surface are found to be less than $5 \%$ in the computational domain and less than $1.5 \%$ at the cylinder surface. Thus, they are within "engineering accuracy". Further in Figure 10 a snapshot at time $t=25 \mathrm{~s}$ for the computed wave fields in the two cases considered. The wave fields have been scaled to fit the figure window. From these figures, it is seen how the local symmetry boundary conditions result in tangential flow to the solid wall faces. 


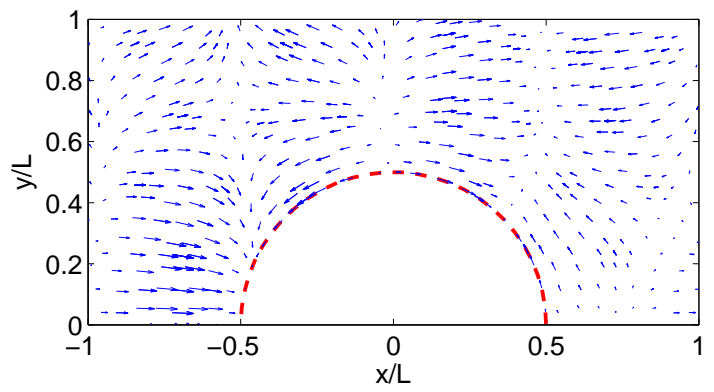

Figure 10. Snapshot of computed wave field in the near-region of the solid surface-piercing cylinder. The cylinder surface is represented using curvilinear approximations.

a)
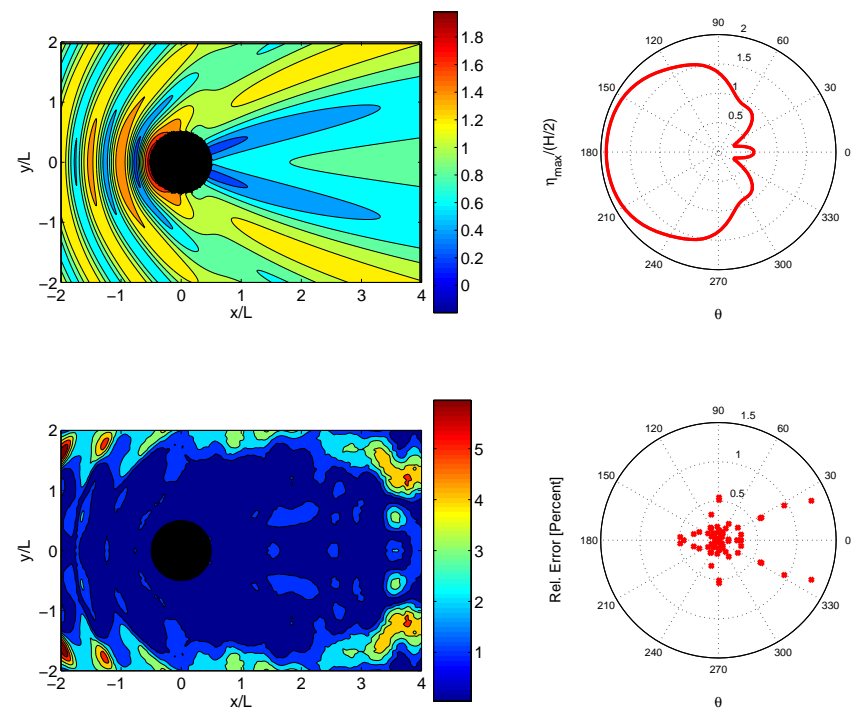

b)

Figure 11. a) Exact, and b) relative errors in percent in computed solution for diffraction and maximum wave run up diagrams for a cylinder in an open water with curvilinear boundary approximations.

\subsection{Regular WaVes over a Semicircular SHOAL}

In a final set of tests, we consider how well the numerical model is able to predict shoaling waves. A linear case if first checked, after which we compare with the experiments carried out by Whalin (1971). It is noted that this test case was also considered in a DG-FEM setting by Eskilsson and Sherwin (2005). 
Following Dingemans (1997) a linear solution of the wave envelope of the refracted wave field can be obtained by introducing a parabolic approximation to the dispersive and non-dissipative mild slope equations resulting in the following equation system accounting for variations in the local wave amplitudes $A(\boldsymbol{x})$

$$
\begin{aligned}
c_{g} & =1+\frac{2 k d}{\sinh (2 k d)}, \\
\partial_{x} A & -i\left(k-k_{0}\right) A+\frac{A}{2 c_{g}} \partial_{x} c_{g}-\frac{i}{2 \omega c_{g}} \partial_{y}\left(c c_{g} \partial_{y} A\right)=0,
\end{aligned}
$$

where the incoming wave field is given in terms of the scalar potential $\phi=A(\boldsymbol{x}) e^{i k_{0} x}$. Here $k_{0}$ is a reference wave number, $k$ the local wave number, and $c_{g}$ the group velocity. This parabolic equation can be solved using a standard Crank-Nicolson finite difference method, e.g. as described in Press et al. (1992).

In the experiments, a wave tank was used containing a semi-circular shoal connecting the deep part of the basin with the the shallow part. The shoal is defined according to (scale in meters)

$$
\begin{aligned}
d(\boldsymbol{x}) & = \begin{cases}0.4572 & , x \leq 10.67-\Lambda(y) \\
d_{\text {shoal }}(\boldsymbol{x}) & , 10.67-\Lambda(y)<x<18.29-\Lambda(y), \\
0.1524 & , x \geq 18.29-\Lambda(y)\end{cases} \\
d_{\text {shoal }}(\boldsymbol{x}) & =0.4572+0.04(10.67-\Lambda(y)-x) \\
\Lambda(y) & =\left\{\begin{array}{ll}
\sqrt{6.096 y-y^{2}} & , y \geq y_{0} \\
\sqrt{6.096 y_{0}-y_{0}^{2}} & , 0 \leq y<y_{0}
\end{array},\right.
\end{aligned}
$$

where we have introduced a parameter $y_{0}$ to be able to exercise some control over the magnitude of the $\partial_{y} d(\boldsymbol{x})$ near $y=0 m$ as very large values in the derivative were found to cause numerical instability at the outer boundaries. Thus, by the minor modification introduced in the shoal definition, we can control the upper limit of the magnitude of the gradient and ensure that it vanishes at the outer boundaries. In the original experiment by Whalin the shoal definition corresponds to the one given with $y_{0}=0 \mathrm{~m}$. Thus, we define $y_{0}=0.01 \mathrm{~m}$ and hence only the southern domain boundary nodes are affected. This allow us to resemble the original experiments closely. The depth contours of the semicircular shoal are shown in Figure 12. Combined wave generation and absorption zones are introduced near the western boundary and have a total length of one and a half wave lengths. At the interface between the relaxation region and the numerical wave tank $(x \approx 5.9$ $\mathrm{m}$ ) the triangle edges coincide with the interfaces of the relaxation zone to obtain a smooth generation, see Figure 13. At the eastern boundary 


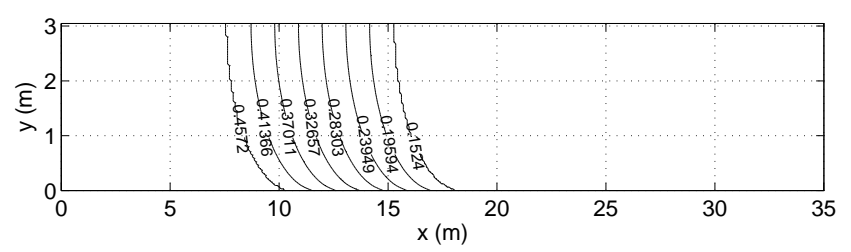

Figure 12. Depth contours of symmetric half of semicircular shoal.

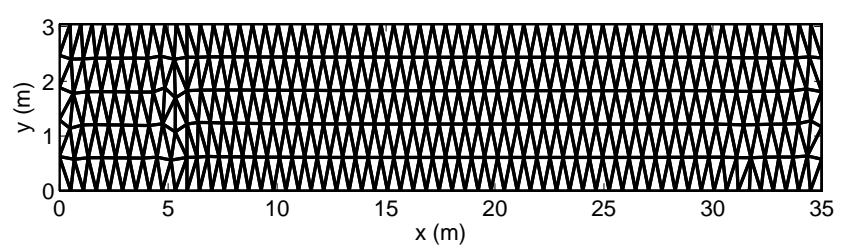

Figure 13. Grid representation of half-sized symmetric part of domain containing 588 elements each of characteristic length approximately $0.6 \mathrm{~m}$. Domain bounding box $\boldsymbol{x} \in[0,35 m] x[0,3.048 m]$.

we absorb outgoing waves over two wave lengths. We conduct a test similar to the original experiment by Whalin (1971) where waves of period $T=2 \mathrm{~s}$ and wave height $H=0.0150 \mathrm{~m}$ are generated in the deep part of the basin. For the linear test we generate a monochromatic progressive wave $(L=3.9095 \mathrm{~m})$ and for a comparison with experimental data we determine a stream function solution $(L=3.91049 \mathrm{~m})$ to avoid harmonic generation due to inconsistent boundary conditions.

In Figure 14 a snapshot of the free surface elevation at $t=50 \mathrm{~s}$ is shown in a linear simulation where $y_{0}=0.01 \mathrm{~m}$. Note, that the solution has been reflected symmetrically about $y=3.048 \mathrm{~m}$ as only half of the domain is used due to the symmetry of the experiment. In the computation a time increment of $\Delta t=0.03 \mathrm{~s}$ was used and a total of 1667 time steps. The spatial domain was subdivided by 588 elements of order $P=4$. The solution method for the linear system was GMRES combined with $\mathcal{L U}$ preconditioning of the linearized system. A comparison between the exact and the computed curve of the wave envelope along the centerline $y=3.048 \mathrm{~m}$ is shown in Figure $15 \mathrm{a}$ ).

In a fully nonlinear simulation we apply a mild exponential cut-off filter with parameters $\left(N_{c}, \alpha, s\right)=(0,12,25)$ for stabilization. A comparison with the experimental data is shown in Figure $15 \mathrm{~b}$ ). The harmonic analysis shows that the amplitudes of the time harmonics are overestimated compared to the experimental data. Further, as a result of some minor Gibbs oscillations in the computation there are noticeable oscillatory patterns in the harmonics. These inaccuracies are unphysical in the sense that they are believed to have been caused by 


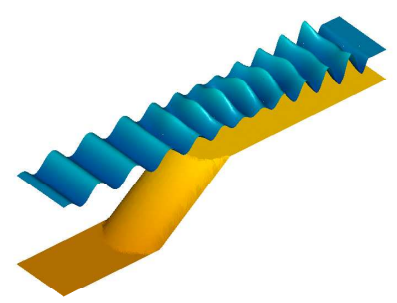

Figure 14. Snapshot at $t=50 \mathrm{~s}$ of free surface in linear refraction test of plane waves over semicircular shoal. Free surface elevation exaggerated 5 times. Padé $(2,2)$ rotational velocity formulation.

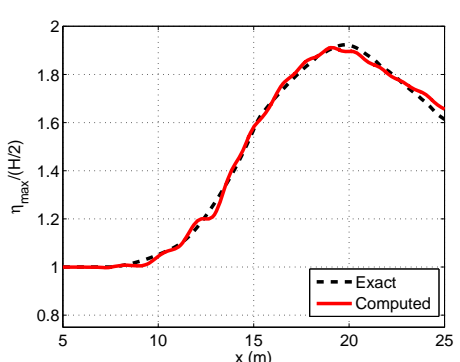

a) Comparison between exact and computed linear solutions along the centerline. $T=2 s$. $P=4$.

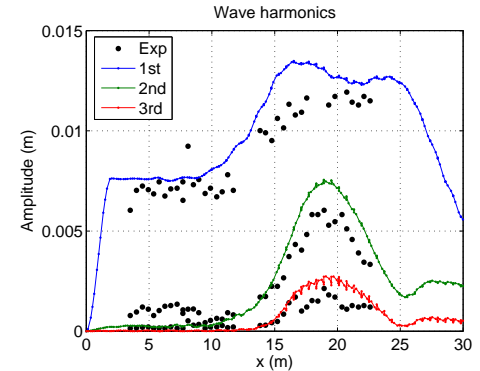

b) Harmonic analysis. Comparison between (nonlinear) experimental and computed solutions along centerline. $T=$ 2s. $P=3$.

Figure 15. Computed linear and nonlinear solutions.

the discontinuous gradients of the depth function $d(\boldsymbol{x})$, which has been computed exactly.

\section{Summary \& concluding remarks}

By the proposed methodology it has been demonstrated by examples in two horizontal dimensions that fundamental and important wavestructure problems can be solved accurately in complex wall domains of arbitrary shapes. Further, the methodology has been kept practical by employing a spatial discretization using a nodal DG-FEM based on the method of Bassi-Rebay. External boundary conditions in a finite domain surrounded by vertical bottom-mounted and surface-piercing walls are straightforwardly imposed using a "ghost"-flow technique combined with simple symmetry considerations. The outcome is a practical method which is suitable for solving a broad range of different 
water wave problems. Current limitations are the efficiency of the model and robustness for nonlinear waves due to aliasing-driven instabilities. These limitations are therefore subject of ongoing research.

\section{Appendix}

\section{A. Differential operators}

A number of differential operators arise as a result of the derivation procedure for the high-order Boussinesq formulation. The definitions of these continuous differential operators for use with either of the three different formulations in two horizontal dimensions are given here for the Padé $(2,2)$ version. The continuous operators are determined using the power rules of $\nabla$ as described in the original work by Madsen et al. (2002). All coefficients and variables used in the definitions are introduced below for the near-optimal choice for the expansion level mid-depth $\sigma=0.5$.

The differential operators for the full rotational velocity formulation are given as

$$
\begin{array}{ll}
\mathcal{A}_{01}=\lambda \partial_{x}+\gamma_{3} \lambda^{3}\left(\partial_{x x x}+\partial_{x y y}\right), & \mathcal{A}_{02}=\lambda \partial_{y}+\gamma_{3} \lambda^{3}\left(\partial_{x x y}+\partial_{y y y}\right), \\
\mathcal{A}_{1}=1-\alpha_{2}\left(\partial_{x x}+\partial_{y y}\right), & \mathcal{A}_{11}=1-\alpha_{2}\left(\partial_{x x}\right), \\
\mathcal{A}_{2}=-\alpha_{2}\left(\partial_{x y}\right), & \mathcal{A}_{22}=1-\alpha_{2}\left(\partial_{y y}\right), \\
\mathcal{B}_{0}=1+\gamma_{2} \lambda^{2}\left(\partial_{x x}+\partial_{y y}\right), & \mathcal{B}_{11}=\beta_{1} \partial_{x}-\beta_{3}\left(\partial_{x x x}+\partial_{x y y}\right), \\
\mathcal{B}_{12}=\beta_{1} \partial_{y}-\beta_{3}\left(\partial_{x x y}+\partial_{y y y}\right), &
\end{array}
$$

with the following operators for handling a mildly varying sea bed

$$
\begin{aligned}
& \mathcal{S}_{01}=\partial_{x} d \cdot \mathcal{C}_{11}+\partial_{y} d \cdot \mathcal{C}_{2}, \quad \mathcal{S}_{02}=\partial_{x} d \cdot \mathcal{C}_{2}+\partial_{y} d \cdot \mathcal{C}_{22}, \\
& \mathcal{S}_{03}=-\partial_{x} d \cdot \mathcal{C}_{13}-\partial_{y} d \cdot \mathcal{C}_{23},
\end{aligned}
$$

where

$$
\begin{array}{ll}
\mathcal{C}_{11}=1-c_{2} \lambda^{2}\left(\partial_{x x}\right), & \mathcal{C}_{2}=-c_{2} \lambda^{2}\left(\partial_{x y}\right), \\
\mathcal{C}_{13}=\lambda \partial_{x}-s_{3} \lambda^{3}\left(\partial_{x x x}+\partial_{x y y}\right), & \mathcal{C}_{22}=1-c_{2} \lambda^{2}\left(\partial_{y y}\right), \\
\mathcal{C}_{23}=\lambda \partial_{y}-s_{3} \lambda^{3}\left(\partial_{x x y}+\partial_{y y y}\right) . &
\end{array}
$$

The local height of the water column from the sea bed to the chosen expansion level is defined as $\lambda \equiv d+\hat{z}$. The spatially varying coefficients are defined as

$$
\begin{aligned}
& \beta_{1} \equiv z-\hat{z}, \quad \beta_{3} \equiv \frac{(z-\hat{z})^{3}}{6}-\frac{\hat{z}^{2}(z-\hat{z})}{10} \alpha_{2} \equiv \frac{(z-\hat{z})}{2}-\frac{\hat{z}^{2}}{10}, \\
& \gamma_{2} \equiv-\frac{2}{5}, \quad \gamma_{3} \equiv-\frac{1}{15} .
\end{aligned}
$$


The free parameter coefficients in the terms responsible for a varying sea bed has been determined for the choice of $\sigma=0.5$ for the Padé $(2,2)$ version in Jamois et al. (2006) to be

$$
c_{2}=0.242027, \quad s_{3}=0.00106481,
$$

minimizing the shoaling errors in the application range $k d \in[0,10]$.

\section{Acknowledgements}

The first author would like to thank Nigel Nuun for helpful improvements of the Sledge ++ software library during the development of the DG-FEM Boussinesq model, and for helpful discussions Prof. Per Madsen from the Coastal, Maritime and Structural Engineering Section, Department of Mechanical Engineering, Technical University of Denmark. The work of the second author was partially supported by the National Science Foundation through the Career Award DMS-0132967.

\section{References}

Ambrosi, D., and Quartapelle, L. A Taylor-Galerkin Method for Simulating Nonlinear Dispersive Water Waves. J. Comp. Phys., Vol. 146, 546-569, 1998.

Antunes Do Carmo, J. S., and Seabra Santos, F. J. Surface Waves propagation in shallow water: A finite element model. J. Numer. Meth. Fluids, Vol. 16, 447-459, 1993.

Bassi, F., and Rebay, S. A High-Order Accurate Discontinuous Finite Element Method for the Numerical Solution of the Compressible Navier-Stokes Equations. J. Comp. Phys., Vol. 131, 267-279, 1997.

Beji, S., and Nadaoka, K. A formal derivation and numerical modelling of the improved Boussinesq equations for varying depth. Ocean Engng., Vol. 23, 691$704,1996$.

Carpenter, M. H., and Kennedy, C. A. Fourth order 2N-storage Runge-Kutta scheme. NASA-TM-109112, NASA Langley Research Center, VA., 1994.

Dingemans, M. W. Water wave propagation over uneven bottoms. World Scientific, 1997.

Engsig-Karup, A.P., Hesthaven, J.S., Bingham, H.B., and Madsen, P. Nodal DGFEM solution of high-order Boussinesq-type equations. J. Engineering Math., To appear, 2006.

Eskilsson, C., and Sherwin, S. J. A Discontinuous Spectral Element Model for Boussinesq-Type Equations. J. Scient. Comp., Vol. 17, 143-152, 2002.

Eskilsson, C., and Sherwin, S. J. An $h p /$ spectral element model for efficient longtime integration of Boussinesq-type equations. Coastal Engineering Journal, Vol. 45, 295-320, 2003.

Eskilsson, C., and Sherwin, S. J. An unstructured spectral/hp element model for enhanced Boussinesq-type equations. submitted to Coastal Engineering Journal, 2005 . 
Eskilsson, C., and Sherwin, S. J. Spectral/hp discontinuous Galerkin methods for modelling 2D Boussinesq equations. J. Comp. Phys., submitted, 2004.

Eskilsson, C., and Sherwin, S. J. Discontinuous Galerkin spectral $/ h p$ element modelling of dispersive shallow water systems. J. Scient. Comp., 22, 279-298, 2005.

Fuhrman, D. R., Bingham, H. B., and Madsen, P. A. Nonlinear wave-structure interactions with a high-order Boussinesq model. Coastal engineering, 52, 655$672,2005$.

Gordon, W. J. and Hall, C. A. Construction of curvilinear coordinate systems and their applications to mesh generation. Int. J. Num. Meth. Engng., Vol. 7, 461-477, 1973.

Hesthaven, J.S. From electrostatics to almost optimal nodal sets for polynomial interpolation in a simplex. SIAM J. Numer. Anal., Vol. 35, No. 2, 655-676, 1998.

Hesthaven, J.S., and Warburton, T. High-Order Nodal Methods on Unstructured Grids. I. Time-Domain Solution of Maxwell's Equations. J. Comput. Phys., 181(1), 186-221, 2002.

Jamois, E., Fuhrman, D. R., Bingham, H. B., and Molin, B. A numerical study of nonlinear wave run-up on a vertical plate. To appear in Coastal Engineering, 2006.

Larsen, J., and Dancy, H. Open boundaries in short wave simulations - a new approach. Coastal Engineering, 7, 285-297, 1983.

Langtangen, H. P., and Pedersen, G. Computational models for weakly dispersive nonlinear water waves. Comput. Methods Appl. Engrg., 160, 337-358, 1998. rt, Department of Mathematical Sciences, Loughborough University.

Li, Y.S, Liu, S.-X., Yu, Y.-X., and Lai, G.-Z. Numerical modelling of Boussinesq equations by finite element method. Coastal engineering, 37, 97-122, 1999.

Madsen, P.A., H.B. Bingham, and H. Liu. A new Boussinesq method for fully nonlinear waves from shallow to deep water. J. Fluid Mech. 462, 1-30, 2002.

Madsen, P.A., Bingham, H.B., and Schäffer, H. A. Boussinesq-type formulations for fully nonlinear and extremely dispersive water waves: derivation and analysis. Proc. R. Soc. Lond. A 459, 1075-1104, 2003.

Madsen, P.A., and Schäffer, H. A. A review of Boussinesq-type equations for gravity waves. In Advances in Coastal and Ocean Engineering, vol 5, 1-95, 1999.

Madsen, P.A., and Sørensen, O. R. A new form of the Boussinesq equations with improved linear dispersion characteristics. Part 2. A slowly varying bathymetry. Coastal Engineering, vol 18, 183-204, 1992.

Madsen, P.A., and Agnon, Y. Accuracy and convergence of velocity formulations for water waves in the framework of Boussinesq theory. J. Fluid Mech., vol 477, 285-319, 2003.

McCamy, R. C. and Fuchs, A. Wave forces on a pile: a diffraction theory. Tech. Memo., No. 69, U.S. Army Board, U.S. Army Corp. of Eng., 1954.

Mei, C.C. The applied dynamics of ocean surface waves. World scientific, 1989.

Nwogu, O. Alternative form of Boussinesq equations for nearshore wave propagation. Journal of Waterway, Port, Coastal and Ocean Engineering, 119 (6), 618-638, 1993.

Panchang, V. G., and Kopriva, D. A. Solution of two-dimensional water-wave propagation problems by Chebyshev collocation. Math. Comput. Modelling, 12, 625-640, 1989.

Penny, W.G. and Price, A. T. Part I. The diffraction theory of sea waves and the shelter afforded by breakwaters. Proc. R. Soc. Lond. A, 244, 236-253, 1952. 
Peregrine, D. H. Long waves on a beach. Journal of Fluid Mechanics, 27, 815-827, 1967.

Persson, P.-O. and Strang, G. A simple mesh generator in Matlab. SIAM Review, 46, 329-345, 2004.

Pos, J.D. A study of breakwater gap wave diffraction using close range photogrammetry and finite and infinite elements. PhD. Thesis. Universitity of Cape Town, South Africa, 1984.

Pos, J.D., and Kilner, F. A. Breakwater gap wave diffraction: An experimental and numerical study. J. Waterway, Port, Coastal and Ocean Eng., ASCE, 113, 1-21, 1987.

William H. Press and Saul A. Teukolsky and William T. Vetterling and Brian P. Flannery Numerical Recipes in C: The Art of Scientific Computing. Cambridge University Press, 1992.

Proriol, J. Sur une famille de polynomes deux variables orthogonaux dans un triangle. C. R. Acad. Sci. Paris, 245, 2459-2461, 1957.

Saad, Y., and Schultz, M. H. GMRES: a generalized minimal residual algorithm for solving nonsymmetric linear systems. SIAM J. Sci. Statist. Comput., 7, 856-869, 1986.

Savitzky, A., and Golay, M. J. E. Smoothening and differentiation of data by simplified least squares procedures. Anal. Chem., 36, 1627-1639, 1964.

Sherwin, S. Dispersion Analysis of the Continuous and Discontinuous Galerkin Formulations. Lecture Notes in Computational Science and Engineering: Discontinuous Galerkin Methods - Theory, Computation and Applications, Springer, 1999.

Skotner, C. and Apelt, C. J. Internal wave generation in an improved twodimensional Boussinesq model. Ocean Engng., 26, 287-324, 1999.

Sommerfeld, A. Mathematische theorie der diffraction. Mat. Ann., 47, 317-374, 1896.

Sørensen, O. R., Schäffer, H. A., and Sørensen, L. S. Boussinesq-type modelling using an unstructured finite element technique. Coastal Engineering, 50, 181-198, 2004.

Walkley, M. A., and Berzins, M. A finite element method for the one-dimensional extended Boussinesq equations. Int. J. Numer. Meth. Fluids., 29, 143-157, 1999.

Walkley, M. A., and Berzins, M. A finite element method for the two-dimensional extended Boussinesq equations. Int. J. Numer. Meth. Fluids., 39, 865-885, 2002.

Whalin, R. W. The limit of applicability of linear wave refraction theory in a convergence zone. Research report H-71-3, U.S. Army Corps of Engineers, WES, Vicksburg, Mi, Res. Rep.

Wilcox, L. High-order accurate methods for solving the time-harmonic Maxwell's equations. Ph.D. thesis, Division of Applied Mathematics, Brown University, USA, 2006. 
paper.tex; 15/12/2006; $9: 52 ;$ p.26 